\title{
O USO DE ANSIOLÍTICO NO PRÉ-ATENDIMENTO EM ODONTOLOGIA - REVISÃO DE LITERATURA
}

\section{THE USE OF ANXIOLYTIC IN DENTISTRY PRE-ATTENDANT - A LITERATURE REVIEW}

\author{
Jessica Leny Gomes Ferreira ${ }^{1}$ \\ Adolfo Saraiva de Miranda Luna ${ }^{1}$ \\ Catarine Santos Rocha ${ }^{1}$ \\ Alessandra Marcondes Aranega ${ }^{2}$ \\ Idelmo Rangel Garcia Júnior ${ }^{2}$ \\ Júlio Maciel Santos de Araújo ${ }^{3}$
}

\section{RESUMO}

A ansiedade produzida perante o procedimento odontológico é um fator que deve ser sempre levado em consideração, principalmente antes de procedimentos cirúrgicos. O profissional deve estar preparado para controlar essa tensão e, dentre as opções existentes, está a utilização de ansiolíticos que promovem sedação consciente, como o Midazolam ou Diazepam. Com relação ao primeiro, um benzodiazepínico, apresenta rápida absorção quando administrado por via oral e extensa margem de segurança. Já o segundo, Diazepam, gera uma sedação de longa duração. Assim sendo, o presente trabalho tem a finalidade de fazer uma revisão da literatura acerca do uso prévio de ansiolítico em Odontologia.

Descritores: Hipnóticos e sedativos; Medicação pré-anestésica; Ansiedade; Medo.

\section{ABSTRACT}

The anxiety produced during dental procedure is a factor that should be taken into consideration, especially before surgical procedures. With the aim of controlling it, in dentistry we have several alternatives, including a choice of using anxiolytic drugs: how Midazolam or Diazepam, which promote conscious sedation. With respect to the first, benzodiazepine, has rapid absorption when administered orally and extensive safety margin. The second, Diazepam, generates a long lasting sedation. Therefore, this study aims to do a review of the literature on previous use of oral anxiolytic in surgery, especially concerning the use of midazolam.

Descriptors: Anxiety; Conscious sedation; Anxiolytics; Midazolam. 
FERREIRA JLG

LUNA ASM

ROCHA CS

ARANEGA AM GARCIA-JÚNIOR IR

ARAÚJO JMS

$O$ USO DE ANSIOLITTICO NO PRÉ-ATENDIMENTO

EM ODONTOLOGIA

- REVISÃO DE LITERATURA

228

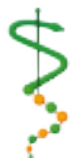

REV. ODONTOL. UNIV. CID. SÃO PAULO

$2014 ; 26(3):$ 227-31, SET-DEZ

\section{N T R O D U ÇÃ O}

Com o passar do tempo, a tecnologia e a invenção de novas técnicas vêm modificando e aprimorando as formas de procedimentos e tratamentos na área da saúde, atrelados ao desejo e busca dos pacientes em obter resultados mais eficientes e eficazes, tanto com relação à função quanto à estética. Porém, no âmbito da Odontologia isso ainda parece distante de muitos pacientes que são permeados pelo medo e ansiedade.

Dentre os variados tipos de medo e fobia, a ansiedade diante dos procedimentos odontológicos é um dos mais frequentemente referidos. Estudo relatou que de $73 \%$ a $79 \%$ dos pacientes alegaram que tiveram pelo menos um pouco de ansiedade no atendimento odontológico ${ }^{1}$. Em outra análise, segundo Malamed² (1996), "ir ao Dentista" foi avaliado como o segundo entre os medos e temores mais frequentes da população. A causa do medo pode estar vinculada a diversos fatores, dentre eles: tratamento doloroso, som da broca, ignorância sobre o que irá acontecer, injeção, entre outros.

A dificuldade em controlar a ansiedade (sentimento vago e desagradável acompanhado por vários tipos de medo e fobia) pode direcionar a um fracasso no tratamento odontológico ${ }^{1}$. É a partir daí que a pré-medicação em procedimentos odontológicos teve indicação relevante, e foi dentro dessa pré-medicação que o enfoque ansiolítico ganhou seu protagonismo particular nessa ciência.

\section{REVISÃO DE LITERATURA}

De acordo com Jankovi et al. ${ }^{3}$ (2014), "a prevalência da ansiedade pré-atendimento grave é relativamente alta". Em um estudo sobre a população urbana de Gotemburgo na Suécia, esse tipo de ansiedade foi encontrado em $6,7 \%$ quando a escala de ansiedade dental com 10 perguntas foi usada. É cerca de quatro vezes maior em pessoas entre 18 e 39 anos de idade do que em pessoas com mais de 60 . Na Noruega foi encontrada entre $11,3 \%$ em homens com 25 anos de idade e 19,8\% em mulheres da mesma idade, mostrando que afeta principalmente pessoas mais jovens, o que demonstra seu caráter negativo sobre a saúde oral. Há muitos fatores potencialmente associados como, por exemplo, em um estudo caso/controle realizado na Dinamarca, a ansiedade odontológica foi associada a medo de perfuração, contatos negativos com dentista e aumento dos sintomas orais.

Sintomas como aumento da pressão arterial, da sensação de dor, aumento da frequência respiratória e da pulsação são intrínsecos à ansiedade gerada pelos procedimentos cirúrgicos na maioria dos pacientes ${ }^{4}$. A utilização da sedação consciente pré-operatória faz o controle dessa barreira na ciência odontológica, possibilitando a respiração de forma normal, depressão ínfima da consciência, onde o paciente mantém o controle de respostas, além de ser coadjuvante na indução da anestesia $^{5,6}$. Essa sedação moderada tem como finalidade promover melhor comodidade e relaxamento para pacientes, conservando a segurança e o simples acesso para profissionais de saúde qualificados. Atualmente, a conciliação de sedação e anestesia local tem sido frequentemente utilizada durante uma variedade de procedimentos cirúrgicos orais, traduzindo-se como necessidade e aumentando substancialmente a demanda por serviços desse nível. Sem dúvida, isso firma uma melhor relação entre o paciente e o Cirurgião-Dentista na ação operatória ${ }^{7}$.

Assim sendo, hoje vivenciamos a procura incessante por um sedativo ideal, que tenha as características de proporcionar amnésia e analgesia, além de ampla margem de segurança, ótima qualidade de sedação, alcançando a satisfação do paciente ${ }^{7,8}$.

Os principais fármacos ansiolíticos utilizados contemporaneamente que possibilitem a sedação moderada são os benzodiazepínicos, em especial o Midazolam e o Diazepam. Segundo Lader ${ }^{9}$ (2008), os benzodiazepínicos a partir dos anos de 1960 passaram a ser muito utilizados no mundo para variados casos nem sempre definidos, como ansiedade, insônia e tensão muscular; porém ainda hoje se constata a problemática de estudos acerca de seus benefícios e malefícios. Ainda assim, 
a indicação dessa classe de drogas no pré-atendimento está ligada aos domínios sedativos, efeito relaxante muscular e produção da amnésia anterógrada.

Seguindo essa linha, muitos estudos têm abordado o Midazolam como o mais utilizado. Conforme Isik et al. ${ }^{10}$ (2008), em doses clínicas corretas ele inibe depressão respiratória ou cardiovascular, e devido à sua eficácia após a consumação oral e à sua extensa margem de segurança, tornou-se popular em Odontologia. Já Horiuchi et al. ${ }^{11}$ (2005) afirmam que uma abundância de medicamentos têm sido prescrita para pré-medicação em crianças, incluindo-se benzodiazepínicos, opioides, barbitúricos e cetamina. Porém, o mais frequentemente administrado como pré-medicação em pediatria nos Estados Unidos da América é o Midazolam, e um grande número de relatórios documentou a sua eficácia por via oral em crianças.

Essa droga supracitada ministra: sedação, amnésia anterógrada e o relaxamento do músculo esquelético com vasto índice terapêutico, e pode ser administrada por uma diversidade de vias ${ }^{7}$. Tem um rápido início de ação, aproximadamente 10 minutos, e curta duração de efeitos categorizados pela meia-vida de eliminação curta do midazolam e do seu metabólito ativo $^{12}$. Gonçalves e Cruz ${ }^{4}$ (2009) completam o pensamento declarando que o Midazolam completa concentração máxima após 30 minutos e tem durabilidade de aproximadamente 2 a 4 horas, devido ao rápido metabolismo. Destaca-se também que, conforme Miyake et al. ${ }^{13}$ (2010) Midazolam pode ser usado não só para sedação, mas também como anestesia geral.

Com relação ao Diazepam, este também apresenta boas características, sendo que sua meia-vida de eliminação é de 24 a 72 horas, se tornando às vezes não interessante para uso em procedimentos odontológicos ${ }^{14}$. Através da análise de Muzyk et al. ${ }^{15}$ (2013) sabe-se que essa droga tem um rápido início de ação e rapidamente atinge a sua concentração máxima no sistema nervoso central, devido a sua natureza altamente lipofílica. Por conseguinte, é metabolizada pelo fígado para formar o metabólito ativo, e o desmetildiazepam e ambos são eliminados lentamente. Essa eliminação lenta permite prolongadas concentrações terapêuticas existirem mesmo após o tratamento de curta duração. Apesar dos efeitos clínicos desaparecerem em 2 a 3 horas, a sonolência e o prejuízo na função psicomotora podem persistir devido à produção de metabólitos ativos ${ }^{16}$.

Através da diversidade de estudos, temos um vasto alicerce sobre a utilização de drogas no pré-operatório. Num estudo feito com pacientes pediátricos observou-se que por via oral a Cetamina pode conferir sedação adequada apenas a 26\% das crianças, indicando que não se obteve tanto sucesso quanto o Midazolam quando administrado por via oral, baseando-se na sedação pré-operatória e facilitação de uma indução anestésica suave e redução da ansiedade. Porém, também alertam que, apesar de ser amplamente recomendado como medicação prévia em crianças, o Midazolam pode não ter sabor agradável, provocar depressão respiratória fatal em casos de overdose e causar efeito paradoxal (confusão, agressividade, resistência e agitação) ${ }^{11,17}$.

Outro estudo, o qual retrata o uso de sedação consciente com benzodiazepínicos na Odontologia, conclui que o Diazepam é recomendado quando se deseja uma sedação pós-operatória mais demorada e que o Midazolam é a droga de escolha para a sedação de pacientes adultos e pediátricos, na maioria dos procedimentos odontológicos, principalmente em casos de urgência, por possuir rápido início de ação e induzir amnésia anterógrada ${ }^{18}$.

Entretanto, é imprescindível destacar também os efeitos negativos dos benzodiazepínicos, insistindo numa maior atenção sobre superdosagem e casos de automedicação contínua. Também evidencia-se a atenção sobre os efeitos adversos como fadiga, ataxia, amnésia e depressão respiratória $^{4,19}$. Com relação ao uso na Odontologia que é de curta duração, promovem baixo índice de toxicidade e de efeitos adversos. É relevante destacar que em uma pequena parcela de pacientes, mesmo em baixas doses, pode ocorrer o efeito paradoxal, o qual é representado pala irritabilidade, excitação e agressividade ${ }^{18}$.
FERREIRA JLG

LUNA ASM

ROCHA CS

ARANEGA AM

GARCIA-JÚNIOR IR

ARAÚJO JMS

O USO DE

ANSIOLITICO NO

PRÉ - ATENDIMENTO

EM ODONTOLOGIA

- REVISÃO DE

LITERATURA

229

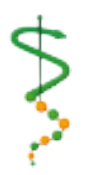

REV. ODONTOL.

Univ, CID. São

PAULO

2014; 26(3):

$227-31$, SET-DEZ 
FERREIRA JLG

LUNA ASM

ROCHA CS

ARANEGA AM GARCIA-JÚNIOR IR

ARAÚJO JMS

O USO DE ANSIOLÍTICO NO PRÉ-ATENDIMENTO EM ODONTOLOGIA - REVISÃO DE LITERATURA

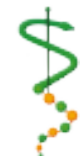

REV, ODONTOL UNIV, C I D, SÃO PAULO 2014; 26(3): $227-31$, SET-DEZ

\section{CONCLUSÃO}

Existem diversas formas de se tranquilizar um paciente no pré-atendimento odontológico. Uma das formas que tem ganho força devido a seu sucesso é o uso de ansiolíticos que irão promover uma sedação consciente, propiciando um aten-

\section{REFERÊNCIAS}

1. Marya CM, Grover S, Jnaneshwar A, Pruthi N. Dental anxiety among patients visiting a dental institute in Faridabad, India. West Indian Med J 2012 Mar;61(2):187-90.

2. Malamed ST. Pain and anxiety control in dentistry. Oral Health 1996 Feb;86(2):11-6.

3. Jankovic SM, Aleksic D, Bahtijari Z, Jelic A, Klacar J, Kovacevic A, et al. Risk factors for severe dental anxiety among medical students. Vojnosanit Preg/ 2014 Jan;71(1):16-21.

4. Gonçalves RDR, Cruz AAV. Midazolam oral como medicação préanestésica em blefaroplastias. Arq Bras Oftalmol 2009 out;72(665-8.

5. Kirova DG, Atanasov DT, Lalabonova CK, Janevska S. Dental anxiety in adults in Bulgaria. Folia Med (Plovdiv) 2010 Apr-Jun;52(2):49-56.

6. Haas DA. Oral and inhalation conscious sedation. Dent Clin North Am 1999 Apr;43(2):341-59.

7. Yen P, Prior S, Riley C, Johnston W, Smiley M, Thikkurissy S. A comparison of fospropofol to midazolam for moderate sedation during outpatient dental procedures. Anesth Prog 2013 Winter;60(4):162-77.

8. Samuelsson K, Pickup K, Sarda S, Swales JG, Morikawa Y, Schulz-Utermoehl T, et al. Pharmacokinetics and metabolism of midazolam in chimeric mice with humanised livers. Xenobiotica 2012 Nov;42(11):1128-37.

9. Lader M. Effectiveness of benzodiazepines: do they work or not? Expert Rev Neurother 2008 Aug;8(8):1189-91. dimento mais tranquilo e adequado tanto para o paciente quanto para o profissional. Devido a suas características, como rápida indução do efeito, efeito de curta duração e causar amnésia anterógrada, o Midazolam é o mais indicado para procedimentos odontológicos.

10. Isik B, Baygin O, Bodur H. Premedication with melatonin vs midazolam in anxious children. Paediatr Anaesth 2008 Jul;18(7):635-41.

11. Horiuchi T, Kawaguchi M, Kurehara K, Kawaraguchi Y, Sasaoka N, Furuya $\mathrm{H}$. Evaluation of relatively low dose of oral transmucosal ketamine premedication in children: a comparison with oral midazolam. Paediatr Anaesth 2005 Aug; 15(8):643-7.

12. Garnock-Jones KP. Oromucosal midazolam: a review of its use in pediatric patients with prolonged acute convulsive seizures. Paediatr Drugs 2012 Aug 1;14(4):251-61.

13. Miyake W, Oda Y, Ikeda Y, Hagihira S, Iwaki H, Asada A. Electroencephalographic response following midazolam-induced general anesthesia: relationship to plasma and effect-site midazolam concentrations. J Anesth 2010 Jun;24(3):386-93.

14. Buffett-Jerrott SE, Stewart SH, Finley GA, Loughlan HL. Effects of benzodiazepines on explicit memory in a paediatric surgery setting. Psychopharmacology (Berl) 2003 Aug;168(4):37786.

15. Muzyk AJ, Leung JG, Nelson S, Embury ER, Jones SR. The role of diazepam loading for the treatment of alcohol withdrawal syndrome in hospitalized patients. Am J Addict 2013 Mar-Apr;22(2):113-8.

16. Khalil SN, Vije HN, Kee SS, Farag A, Hanna E, Chuang AZ. A paediatric trial comparing midazolam/Syrpalta mixture with premixed midazolam syrup (Roche). Paediatr Anaesth 2003 Mar;13(3):205-9. 
17. Loeffler PM. Oral benzodiazepines and conscious sedation: a review. J Oral Maxillofac Surg 1992 Sep;50(9):98997.

18. Volpato MC, Cogo K, Bergamaschi CC, Yatsuda R, Dias AE. Sedação consciente com benzodiazepínicos em odontologia. Rev Odontol Univers Cidade de São Paulo 2006 maio-ago;18(2):181-8.
19. Smiley MK, Prior SR. Dexmedetomidine sedation with and without midazolam for third molar surgery. Anesth Prog 2014 Spring;61(1):3-10.

Recebido em 21/01/2015

Aceito em 11/03/2015
FERREIRA JLG

LUNA ASM

ROCHA CS

ARANEGA AM

GARCIA-JÚNIOR IR

ARAÚJO JMS

O USO DE

ANSIOLÍTICO NO

PRÉ-ATENDIMENTO

EM ODONTOLOGIA

- REVISÃO DE

LITERATURA

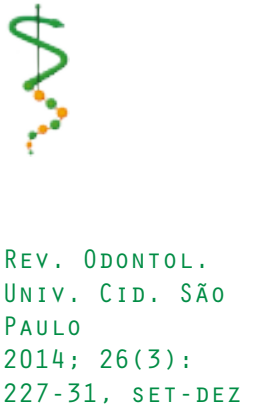

